\title{
24. Sur quelques points de la théorie du potentiel (II).
}

\author{
Par Kinjiro KunUgI. \\ Institut de Mathématiques, Université Impériale de Hokkaido. \\ (Comm. by T. Takafi, M. I. A., Oct. 13, 1947.)
}

$\S 1$. Définition de la capacité des ensembles. Pour fixer les idées, nous nous bornnerons au cas de l' espace à trois dimensions, qui sera désigné par $\omega$. Soient $F$ un ensemble fermé et borné situé dans $\omega$, et $\mu$ une fonction d'ensembles, complètement additive, determinée par une distribution de masse non-négatiue, répartie sur $F$. Dans la partie $(\mathrm{I})^{1)}$, nous avons introduit des potentiels généralisés définis comme il suit : Soit $\Phi(t)$ une fonction réelle, définie dans l'intervalle $0<t<+\infty$ et mesurable au sens de Lebesgue, et posons ${ }^{2)}$

$$
u(p)=u(p, \mu)=\int_{F} \Phi\left(\frac{1}{r_{p q}}\right) d \mu_{q}
$$

où $p$ est un point quelconque de $\omega, q$ un point variable dans $F$ et $r_{p q}$ désigne la distance euclidienne de $p$ à $q$.

Introduisons d'abord la

Condition $(\alpha)$ : $\Phi(t)$ est une fonction monotone croissante et convexe. Dans les considérations ultérieures, ommettons les cas où $\lim _{t \rightarrow \infty} \Phi(t)<+\infty$. puisque $\Phi(t)$ y se réduit à une constante. Donc, on a toujours $\lim \Phi(t)=+\infty$, et l'intégrale de la défiinition (1) sera donnée comme intégrale impropre au sens de Lebesgue.

Nous avons démontré dans la partie (I) que les porentiels généralisés définis en vertu de (1) jouissent, dans la condition $(\alpha)$, des propritétés suivantes :

$\left.1^{\circ}\right) u(p)$ est continue dans $\omega-F$, et semi-continue inférieurement partout dans. $\omega$.

$\left.2^{\circ}\right) \quad u(p)$ est subharmonique dans $\omega-F$.

$\left.3^{\circ}\right) u(p)$ satisfait au principe $d u$ maximum: Soient $D$ un des domaines connexes en lesquels se décomposent l'ensemble complémentaire de $F$ et $H$ la frontière de $D$. Si l'on a linégalité : $u(p) \leq K$ (où $K$ est une constante), pour tout point $p$ de $H$, la même inégalité subsiste partout dans $D$.

Pour fixer les idées, introduisons encore une hypothèse faible:

Condion $(\beta)$ : nous avons $\lim _{t \rightarrow 0} \Phi(t)=0$.

Puisque $\Phi(t)$ est monotone croissante, on a, d'après $(\beta), \Phi(t) \geq 0$ pour $0<t<+\infty$. Maintenant, définissons la notion de capacité des ensembles selon M. de la Vallee Poussin ${ }^{3}$. Soit $A$ un ensemble borelien situé

1) K. Kunugui: Sur quelques points de la théorie du potentiel (I). présenté par M. T. Takagui, M. I. dans la séance du 12 Mars 1945.

2) O. Frostman: Potentiel d'équilibre et capacité des ensembles avec quelques applications à la théorie des fonctions, Thèse, Lund, 1935, p. 17.

3) Ch. de la Vallée Poussin: Les nouvelles méthodes de la théorie du potentiel et la problème généralise de Dirichlet, Paris, 1937, p. 19. 
dans $\omega$. Alors, la borne supérieure de la masse totale $\mu(A)$ de toutes les distributions de masse non-négative, répartie sur tout sous-ensemble $F$ fermé et borné de $A$, tels qu'on ait $u(p, \mu) \leq 1$ partout dans $\omega(F$ et $\mu$ étant variables), s'appelle la capacité de $A$ et sera désignée par $C(A)$.

Il est manifeste que la eapacité est une fonction d'ensembles nonnégative qui jouit de deux propriétés suivantes, caractéristiques à la mesure extérieure: (I) pour deux ensembles quelconques $M, N$ tels que $M \supseteq N$, nous avons $C(M) \geq C(N)$; (II) pour toutes les suites d'ensembles $A_{n}(n=1,2.3, \ldots \ldots)$, nous avons

$$
C\left(\sum_{\infty}^{\infty} A_{n}\right) \leq \sum_{n=1}^{\infty} C\left(A_{n}\right)
$$

D'autre part, il faut remarquer que, pour que la capacité $C(A)$ ne soit identiquement nulle, il faut et il suffit que $\Phi(t)$ satisfasse à la

$$
\text { Condition }(\gamma): \quad \int_{0}^{1} \Phi\left(\frac{1}{\xi}\right) \cdot \xi^{2} d \xi<+\infty \text {. }
$$

Supposons donc $(\gamma)$. Or, en vertu de $(\gamma)$, nous pouvons dire que (III) l'ensemble de capacite nulle ne peut porter aucune masse, en particulier, il est de mesure nulle au senu de Lebesgue.

§2. Théoreme fondamental. Considérons maintenant le problème de la distribution d'équilibre :

Theorème ${ }^{1)}$. Supposons que $\Phi(t)$ satisfasse aux conditions $(\alpha),(\beta)$ et $(\gamma)$. Soit $F$ un ensemble fermé et borné quelconque, situé dans $\omega$ et de capacité positive. Il existe alors une distribution de masse $\mu^{*}$ non-négative, d'unité au total, répartie sur $F$ et qui jouit de la propriété : le potentiel engendré par $\mu^{*}$ est constante et égal a som maximum en tout point de $F$ sauf au plus dans un ensemble de capacité nulle. D'ailleurs, la distribution de la masse ayant cette propriété, dite distribution d'équilibre, est unique.

Nous allons esquisser une démonstration de l'existence de le distribution d'équilibre. Son unicité sera démontree en détail dans le $\S 4$. Soit $\mu$ une distribution de masse non-négative, répartie sur $F$ et telle que $\mu(F)=1$. Il en existe une dont le potentiel $u(p, \mu)$ est borné. Cela est une conséquence immédiate de la définition de capacité selon M. de la Vallee Poussin. Donc, la borne inférieure $V$ de l'intégrale d'énergie :

$$
I(\mu)=\int_{F} u(p, \mu) d \mu_{p}
$$

est un nombre réel fini et non-négatif. Il existe alors une distribution $\mu^{*}$, répartie sur $F$ et pour laquelle on a $\mu^{*}(F)=1$ et $I\left(\mu^{*}\right)=V$. La masse $\mu^{*}$ fournit alors une solution de notre problème. Pour le voir, nous re marquons d'abord que le potentiel $u\left(p, \mu^{*}\right)$ satisfait à l'inégalité $u\left(p, \mu^{*}\right) \geq V$ en tout point de $F$ sauf d'un ensemble de capacité nulle. En effet, si l'ensemble $E$ de tous les points de $F$ où l'on a $u\left(p, \mu^{*}\right)<V$ est de capacité positive, nous pouvons appliquer le principe de Gauss, de sorte qu'on soit amené à une contradiction. 
D'autre part, comme $u\left(p, \mu^{*}\right)$ est semi-continue inférieurement et que $I\left(\mu^{*}\right)=V, u\left(p, \mu^{*}\right)$ est nécessatrement $\leq V$ partout dans le noyau de $\mu^{*}$, et le principe du maximum montre bien que $u\left(p, \mu^{*}\right) \leqq V$ partout dans $\omega$. c. q. f. d.

$\S 3$. Intégrale d'énerigie. Considérons maintenant une fonction d'ensembles $\sigma$ complètement additive, engendrée par une masse de signe variable. Nous suposons que sa variation totale est bornée. Donc nous pouvons mettre $\sigma=\mu-\nu$, où $\mu, \nu$ sont deux distributions de mosse non-négative. Nous posons, comme d'habitude, $u(p, \sigma) \equiv$ $u(p, \mu)-u(p, \nu)$. Nous appelons

$$
I(\sigma)=\int_{F} \int_{F}^{\bullet} \Phi\left(\frac{1}{r_{p q}}\right) d \sigma_{p} d \sigma_{q}
$$

“intégrale d'énergie." De cette intégrale, démontrons ${ }^{1)}$

Premier theorème ; Pour toute fonction d'ensembles, complètement additive $\sigma$, l'intégrale d'énergie $I(\sigma)$ est toujours $\geqq 0$.

Deuxième theorème: Si $I(\sigma)=0$, alors $\sigma$ s'annule identiquement.

Démonstration du premier théorème. Désignons par $x_{1}, x_{2}, x_{3}$ et $y_{1}, y_{2}, y_{3}$ trois coordonnées du point $p$ et $q$ respectivement, et posons

$$
\xi_{i}=x_{i}-y_{i}, \quad i=1,2,3 .
$$

Nous avons alors $r=r_{p q}=\sqrt{ } \Sigma_{i} \xi_{i}^{2}$. D'autre part, comme $F$ est borne le diamètre $\delta(F)$ de $F$ est fini. Soit $M$ un nombre réel fini quelconque, supérieur à $\delta(F)$. Posons $h(t)=0$ ou 1 suivant que $0<t<1 / M$ ou $1 / M$ $\leqq t<+\infty$, et $\Phi^{*}(t)=\Phi(t) \cdot h(t)$.

Considérons maintenant le transformé de Fourier de la fonction $\Phi *(1 / r)$ :

$$
\begin{aligned}
E\left(\alpha_{1}, \alpha_{2}, \alpha_{3}\right) & =\left(\frac{1}{2 \pi}\right)^{3} \iint_{-\infty}^{+\infty} \int \Phi^{*}(1 / r) e^{-i \Sigma \alpha_{k} \xi_{k}} d \xi_{1} d \xi_{1} d \xi_{3} \\
& =\left(\frac{1}{2 \pi}\right)^{3} \iint_{-\infty}^{+\infty} \int \Phi^{*}(1 / r) \cos \left(\sum_{k=1}^{3} \alpha_{k} \xi_{k}\right) d \xi_{1} d \xi_{2} d \xi_{3} \\
& =\frac{1}{2 \pi^{2} \alpha} \int_{0}^{\infty} r \Phi^{*}(1 / r) \sin \alpha r d r^{2)},
\end{aligned}
$$

oû 1' on pose $\alpha=\sqrt{ } \Sigma \alpha_{k}{ }^{3}$. En vertu de $(\gamma)$, l'intégrale simple existe toujours, puisqu' on ait $\left|\boldsymbol{r}^{*}(1 / r)\right| \leqq \alpha r^{2} \Phi(1 / r)$, et $\Phi^{*}(1 / r) \equiv 0$ pour $M<r$.

Or, puisque $\Phi(t)$ est monotone croissante et convexe et que $\lim \Phi(t)=0, \Phi(t) / t$ est monotone croissante pour $0<t<+\infty$. La fonction $r \Phi^{*}(1 / r)$ est donc monotone décroissante pour $0<r<+\infty$. Par consequent le transforé de Fourier $E\left(\alpha_{1}, \alpha_{2}, \alpha_{3}\right)$ est positive, sauf le cas où $\alpha=M /(2 m \pi)(=1,2,3, \ldots \ldots)$ et de plus $r \Phi^{*}(1 / r)$ est constante pour $0<r<M$. Dans ce cas exceptionel, on a $E\left(\alpha_{1}, \alpha_{2}, \alpha_{3}\right)=0$.

1) Cf. Frostman, loc. cit. p. 28-33.

2) Pour la déduction, voir p. ex. Bochner: Vorlesungen uber FourieIsche Integrale, Leipzig, I932, p. 187. 
Si $\Phi^{\prime \prime}(t)$ existe et est continue, on $\mathrm{a}^{1)}$

$$
\begin{aligned}
\Phi^{*}(1 / r) & =\lim _{n \rightarrow \infty} \iint_{-n}^{+n} \int E\left(\alpha_{1}, \alpha_{2}, \alpha_{3}\right) e_{k}^{i \Sigma x_{k} \xi_{k}} d \alpha_{1} d \alpha_{2} d \alpha_{3} \\
& =\lim _{n \rightarrow \infty} \iint_{-n}^{+n} \int E\left(\alpha_{1}, \alpha_{2}, \alpha_{3}\right) \cos \left(\sum_{k=1}^{3} \alpha_{k} \xi_{k}\right) d \alpha_{1} d \alpha_{2} d \alpha_{3},
\end{aligned}
$$

et d'après (2)

$$
\begin{aligned}
\Phi^{*}(1 / r) & =\lim _{n \rightarrow \infty} \iint_{-n}^{+n} \int E\left(\alpha_{1}, \alpha_{2}, \alpha_{3}\right) \cos \left(\sum_{k} y_{k} \alpha_{k}\right) \cos \left(\sum_{k} y_{k} \alpha_{k}\right) d \alpha_{1} d \alpha_{2} d \alpha_{3} \\
& +\lim _{n \rightarrow \infty} \iint_{n}^{+n} \int E\left(\alpha_{1}, \alpha_{2}, \alpha_{3}\right) \sin \left(\Sigma x^{k} \alpha_{k}\right) \sin \left(\sum y_{k} \alpha_{k}\right) d \alpha_{1} d \alpha_{2} d \alpha_{3} .
\end{aligned}
$$

Les limites dans ces formules sont des convergences uniformes dans l'intérieur de l'intervalle $0<\boldsymbol{r}<\boldsymbol{M}$. Enfin, nous pouvons poser

$$
\begin{gathered}
I(\sigma)=\int_{F} \int_{F} \Phi^{*}\left(1 / r_{p q}\right) d \sigma_{p} d \sigma_{q}=J_{1}+J_{2}, \\
J_{1}=\int_{F} \int_{F} d \sigma_{p} d \sigma_{q} \lim _{n \rightarrow \infty} \int_{0}^{+n} \int_{-n} \int E\left(\alpha_{1}, \alpha_{2}, \alpha_{3}\right) \cos \left(\sum_{k} x_{k} \alpha_{k}\right) \cos \left(\sum_{k} y_{k} \alpha_{k}\right) d \alpha_{1} d \alpha_{2} d \alpha_{1}, \\
J_{2}=\int_{F} \int_{F} d \sigma_{p} d \sigma_{q} \lim _{n \rightarrow \infty} \iint_{-n}^{+n} \int E\left(\alpha_{1}, \alpha_{2}, \alpha_{3}\right) \sin \left(\sum_{k} x_{k} \alpha_{n}\right) \sin \left(\sum_{k} y_{n} \alpha_{k}\right) d \alpha_{1} d \alpha_{2} d \alpha_{3} .
\end{gathered}
$$

Les limites dans formules étant uniformes sauf pour le cas où $r=0$, l'ordre de l'intégaation peut être permutée :

$$
\begin{aligned}
J_{1} & =\lim _{n \rightarrow \infty} \iint_{-n}^{+n} \int E\left(\alpha_{1}, \alpha_{2}, \alpha_{3}\right) \int_{F} \cos \left(\sum_{k} x_{k} \alpha_{k}\right) d \sigma_{p} \int_{i} \cos \left(\sum_{k} y_{k} \alpha_{k}\right) d \sigma_{q} d \alpha_{1} d \alpha_{2} d \alpha_{3} \\
& =\lim \iint_{-n}^{+n} \int E\left(\alpha_{1}, \alpha_{2}, \alpha_{3}\right) \cdot\left\{\int_{Y} \cos \left(\sum_{k} x_{k} \alpha_{k}\right) d \sigma\right\}^{3} d \alpha_{1} d \alpha_{2} d \alpha_{3} \geqq 0
\end{aligned}
$$

De même, nous avons $J_{2} \geq 0$. Par suite $I(\sigma) \geq 0$.

Dans le cas général (où p. ex. $\Phi^{\prime \prime}(t)$ n'existe pas), il est manifeste qu'on peut choisir une suite des fonctions : $\Phi_{n}(t), n=1,2,3, \ldots \ldots$, ayant la seconde dérivée $\Phi_{n}{ }^{\prime \prime}(t)$ continue, satisfaisant aux conditions $(\alpha),(\beta)$, $(\gamma)$ et qui converge vers $\Phi(t)$ uniformément dans l'intervalle $0<t<+\infty$.

On a alors

$$
I(\sigma)=\lim \iint \Phi_{n}\left(1 / r_{p q}\right) d \sigma_{\nu} d \sigma_{q}
$$

et dans ce cas aussi, nous avons $I(\sigma) \geq 0$.

Démonstration du deuxième théorème. Dans le cas où $I(\sigma)=0$, la suite $E_{n}\left(\alpha_{1}, \alpha_{2}, \alpha_{3}\right)$ des transformes de Fourier de $\Phi_{n}^{*}(1 / r)=\Phi_{n}(1 / r) \cdot h(1 / r)$ convergeant uniformément vers $E\left(\alpha_{1}, \alpha_{2}, \alpha_{3}\right)$, nous avons, pour presque tout $\left(\alpha_{1}, \alpha_{2}, \alpha_{3}\right)$

1) Cf. Bochner. loc. cit. p. 202. 


$$
\int_{\psi^{\prime}}\left\{\cos \left(\sum_{k} x_{k} \alpha_{k}\right)\right\} d \sigma_{p}=0, \int_{\psi^{\prime}}\left\{\sin \left(\sum_{k} x_{k} \alpha_{k}\right)\right\} d \sigma_{p}=0 .
$$

Or, supposons par impossible que le fonction $\sigma$ ne soit pas identiquement nulle. Puisque toutes les distributions de masse sont déterminées par leurs valeurs pour les sphères, il existe une sphère $S$ telle qu'on ait soit $\sigma(S)>0$ soit $\sigma(S)>0$. Sans restreindre la généralité, nous pouvons supposer que c'est le premier cas qui a lieu et que la surface de $S$ ne porte aucune masse de $\sigma$. Soit $f(p)$ une fonction de point $p, p \epsilon \omega$, définie comme il suit : $f(p)=1$ ou 0 suivant que $p \in S$ ou non. Désignons par $g\left(\alpha_{1}, \alpha_{2}, \alpha_{3}\right)$ le transformé de Fourier de $f(p)$. Nous avons alors, pour tous les points $p$, excepté ceux de la surface de $S$

$$
f(p)=\iint_{-\infty}^{+\infty} \int g\left(\alpha_{1}, \alpha_{2}, \alpha_{3}\right) e^{i \sum \alpha_{k} x_{k}} d \alpha_{1} d \alpha_{2} d \alpha_{3}
$$

où $x_{1}, x_{2}, x_{3}$ désignent les coordonnées du point $p$. Alors, d'une part, en vertu de la définition de $f(p)$, on a

$$
\int_{r} f(p) d \sigma_{n}=\int_{\mu . S} d \sigma_{p}=o(S)>0,
$$

et d'autre part, d'après (4) et (5), on a

$$
\begin{aligned}
\int_{F^{\prime}} f(p) d \sigma_{p} & =\int\left\{\iint_{F}^{+\infty} \int_{-\infty}^{+\infty} g\left(\alpha_{1}, \alpha_{2}, \alpha_{3}\right) e^{i \sum_{k} x_{k} \alpha_{k}} d \alpha_{1} d \alpha_{2} d \alpha_{3}\right\} d \sigma_{p} \\
& =\iint_{-\infty}^{+\infty} g\left(\alpha_{1}, \alpha_{2}, \alpha_{3}\right) \cdot\left\{\int_{F^{*}} e^{i \sum_{k} x_{k} \alpha_{k}} d \sigma_{p}\right\} \cdot d \alpha_{1} d \alpha_{2} d \alpha_{3}=0 .
\end{aligned}
$$

Ainsi, nous sommes amenés à une contradiction.

§4. Unicité de la distribution de masse d'équilibre. Maintenant qu'avoir disposé tous ceux qui sont nécessaires, nous sommes en état de prouver l'unicité de la solution de problème fondamental ${ }^{12}$. En effet, en vertu de la propriété (III) de capacité, nous avons $u\left(p, \mu^{*}\right)$ $=V$ partout dans $F$ sauf un ensemble de mesure nulle. Or, s'il existe deux distributions de masse $\mu_{1}, \mu_{2}$ qui engendre des potentiels d'équilibre, posons $\sigma=\mu_{1}-\mu_{2}$. Nous avons ainsi une fonction complètement additive, à variation bornée et telle que

$$
I(\sigma)=\int_{F}\left\{u\left(p, \mu_{1}\right)-u\left(p, \mu_{2}\right)\right\} d\left(\mu_{1}-\mu_{2}\right)=0 .
$$

Par suite, d'après le deuxième théorème de l'intégrale d'énergie, nous avons $\sigma \equiv 0$ ou $\mu_{1} \equiv \mu_{2}, c . q . f$. $d$.

$\S 5$. capacité selon M. Frostman ${ }^{2}$. Considérons maintenant le cas où la

Condition $(\delta): \Phi(t) \neq 0$ pour $0<t<+\infty$, est remplie. Dans ce cas, en vertu de $(\alpha)$ et $(\beta), \Phi(t)$ est une fon-

1) Frostman, loc. cit. p. 38.

2) Frostman, loc. cit. p. 49. 
ction croissante au sens strict. Posons de plus $\Phi^{-1}(0)=0$, et définissons "capacité $c^{*}(A)$ de l'ensemble $A$ " par l'équation :

$$
\Phi\left(c^{*}(\boldsymbol{A})\right)=c(A) \text {. }
$$

La capacité $c^{*}(A)$ satisfait évidemment aux proprietes (I) et (III), mais nous pouvons voir qu'elle jouit encore de (II). Pour ce but, remarquons d'abord que toute fonction $\varphi(t)$ définie pour $0 \leq t \leq+\infty$, monotone croissante et concave, et telle que $\phi(0)=0$, satisfait $\dot{a}^{10}$

$$
\varphi\left(\sum_{n=1}^{\infty} t_{n}\right) \leq \sum_{n=1}^{\infty} \varphi\left(t_{n}\right) \text {, pour } 0 \leq t_{n} \leq+\infty, n=1,2,3 \ldots \ldots
$$

En effet, l'inégalité $t_{i} \leq \sum_{b=1}^{\infty} t_{n}(t=1,2,3, \ldots \ldots)$ entraine

$$
\varphi\left(t_{i}\right) \geq \varphi\left(\sum_{n=1}^{\infty} t_{n}\right) \cdot t_{i} /\left(\sum_{n=1}^{\infty} t_{n}\right), i=1,2,3, \ldots \ldots
$$

Et, l'addition par rapport aux indices $i$ nous donne immédiatement l'inégalité (6). Or, $\Phi(t)$ étant croissante et convexe, $\Phi^{-1}$ sera croissante et concave. On a également $\lim \Phi^{-1}(t)=0$. Ainsi, nous pouvons nous servir de (6) en posant $\varphi(t)=\Phi^{-1}(t), t_{n}=c\left(A_{n}\right)$, et obtenons

$$
\Phi^{-1}\left(\sum_{n=1}^{\infty} c\left(A_{n}\right)\right) \leq \sum_{q=1}^{\infty} \Phi^{-1}\left(c\left(A_{n}\right)\right)=\sum_{n=1}^{\infty} c^{*}\left(A_{n}\right) .
$$

D'autre part, $\Phi^{-1}$ étant croissante, nous avons

$$
\Phi^{-1}\left(\sum_{n=1}^{\infty} c\left(A_{n}\right)\right) \geq \Phi^{-1}\left(c\left(\sum_{n=1}^{\infty} A_{n}\right)\right)=c^{*}\left(\sum_{n=1}^{\infty} A_{n}\right)
$$

d'où l'inégalité $c^{*}\left(\sum_{n=1}^{\infty} A_{n}\right) \leqq \sum_{n=1}^{\infty} c^{*}(A), c . q . f . d$.

$\S 6$. Exemples. À part du potentiel newtonien, nous pouvons considérer les potentiels généralisés d'ordre $\kappa^{4)}$ :

$$
\Phi(t)=t^{\kappa}, 1 \leq \kappa<3 \text {. }
$$

$\kappa=1$ est le cas de newtonien. D'autre part, nous pouvons poser ${ }^{5)}$

$$
\Phi(t)=t \cdot e^{-\lambda t}, 0 \leq \lambda<+\infty
$$

Si $\lambda=0$, le potentiel se réduit encore au newtonien. Tous ces $\Phi(t)$ satisfont évidemment à quatre conditions $(\alpha),(\beta),(\gamma)$ et $(\delta)$.

1) Cf. Pringsheim: Zur Theorie der ganzen transcendenten Functionen, Sitzungsber. d. math. phys. Classe d. k. bayer. Akad. d. W., Bd. 32, 1902, p. 170, et Nachtrag, ibid. p. 299. Jensen: Sur lcs fonctions convexes et les inégalités entre les valeurs moyennes, Acta Math. t. 30, 1906, p. 192.

2) Frostman, loc. cit. p. 20.

3) Cf. Seeliger : Ueber das Newtonsche Gravitationsgesetz, Sitzungsber. d. math. phys. Classe d. k. bayer. Akad. d. W,: Bd. 26, 1896, p. 373. H. Yukawa : On the interraction of elementary particles, I, proc. Phys.-Math. Soc. Japan, vol. 17, 1935, p. 48. 